\title{
Ärztenetze in der Schweiz 2010 - auf dem Sprung zu Integrierter Versorgung
}

Peter Berchtold, Christian Peier, Karen Peier

Korrespondenz:

Dr. med. Peter Berchthold College-M

Postfach

CH-3000 Bern 16

peter.berchtold@college-m.ch
Seit 20 Jahren wird in der ambulanten Versorgung der Schweiz vernetzt, häufig gemeinsam, von Ärzten und Versicherern initialisiert und vorangetrieben. Dieses Miteinander sich ansonsten skeptisch gegenüberstehender Akteure gilt als ein wesentlicher Erfolgsfaktor der Schweizer Managed-Care-Geschichte. Das Forum Managed Care verfolgt und publiziert seit vielen Jahren die Entwicklung der Ärztenetze und HMO in der Schweiz [1]. Die neusten Zahlen der Erhebung 2010 belegen das eindrückliche Wachstum und das Potential für die Entwicklung der Integrierten Versorgung.

\section{Nationale Erhebung}

Die Studie wird seit 10 Jahren in einjährigem bzw. zweijährigem Rhythmus durch «College-M» im Auftrag des Forum Managed Care durchgeführt. Die Angaben werden von sämtlichen Ärztenetzen in der Schweiz bzw. deren Betriebsgesellschaften mittels Online-Fragebogen und Direktansprachen erhoben. In die Erhebung eingeschlossen werden alle jene Netze, die mit einem oder mehreren Krankenversicherern einen Vertrag abgeschlossen haben. Die vorliegende Publikation fasst die wichtigsten Resultate der Erhebung 2010 zusammen. Die vollständigen Daten bzw. die Angaben der einzelnen Netze sind über die Webseite des Forum Managed Care verfügbar [2].

\section{Abbildung 1}

Entwicklung der Anzahl Versicherter in Ärztenetzen pro Kanton und ganze Schweiz 2000-2010.

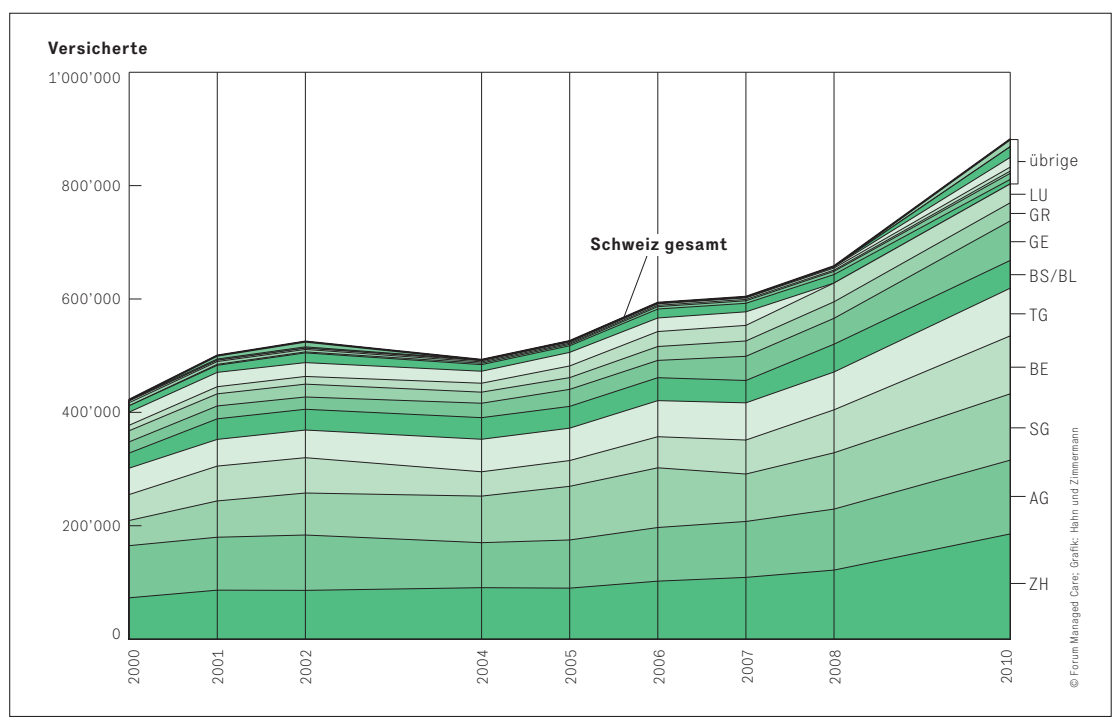

\section{Abbildung 2}

Erwartungen zur zukünftigen Entwicklung der Versichertenzahlen in der Integrierten Versorgung in den nächsten 3 Jahren (Steigerung in \% pro Jahr).

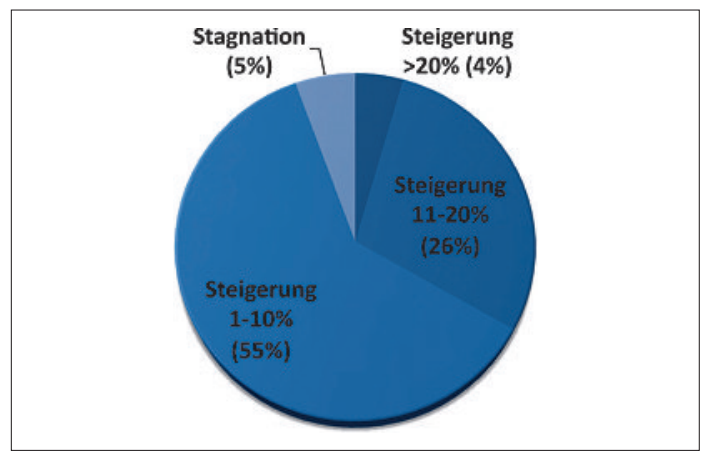

\section{Zwölf bis fünfunddreissig Prozent der Versicherten}

Durchschnittlich jeder achte, im Kanton Thurgau jeder dritte Versicherte entscheidet sich heute für eine hausärztliche Betreuung in einem Ärztenetz, d.h. in einer Organisation, die durch vertraglich vereinbartes Zusammenwirken unter sich, mit netzfremden Leistungserbringern und mit den Versicherern auf die Bedürfnisse der Patientinnen und Patienten ausgerichtete Gesundheitsleistungen erbringt. Gegenüber 2008 ist das ein Zuwachs von 34\%, wobei der Kanton Zürich mit einem Zuwachs von 52\% an der Spitze liegt, gefolgt von den Kantonen Bern (35\%), Thurgau (26\%), Aargau (21\%) und St. Gallen (18\%) (Abb. 1). In den Netzen ist man überzeugt, dass dieses Wachstum anhält: $55 \%$ der Befragten erwarten eine Steigerung der Versicherten von bis zu 10\% jährlich, 26\% der Befragten sogar eine solche von bis zu 20\% (Abb. 2).

\section{Sechsundachtzig Ärztenetze und fünfzig Prozent der Grundversorger}

Wir zählen zurzeit 86 Ärztenetze: Abbildung 3 zeigt ihre geographische Verteilung und die Anzahl ihr angeschlossener Ärzte, Abbildung 4 illustriert die Grösse der Ärztenetze anhand der im Netz betreuten Versicherten sowie das Netzalter nach Betriebsjahren. Die nordostschweizerischen Regionen sowie die Kantone Bern, Genf und Aargau zeigen einen überdurchschnittlichen Anteil an integrierter ambulanter Versorgung: Im Kanton Thurgau haben sich rund 35\% der Versicherten für diese Form entschieden, im Kan- 
ton St. Gallen sind es 25\%, im Aargau 22\%, in Genf $17 \%$ und Zürich $14 \%$ der Versicherten. (Noch) keine Ärztenetze finden wir in den Kantonen Glarus, Jura, Obwalden, Uri, Waadt und Wallis, wobei in einigen dieser Regionen entsprechende Projekte am Start stehen oder von überregionalen bzw. gesamtschweizerischen Netzorganisationen bedient werden. Den 86 Ärztenetzen haben sich rund 50\% aller Grundversorger (Allgemeinmedizi-ner, Internisten, Pädiater) und über 400 Spezialärzte angeschlossen. Dreiundsiebzig der 86 Netze (84\%) haben in ihren Verträgen eine Budgetmitverantwortung

\section{Abbildung 3}

Alle Ärztenetze in der Schweiz mit Anzahl der im Netz angeschlossenen Ärzte.

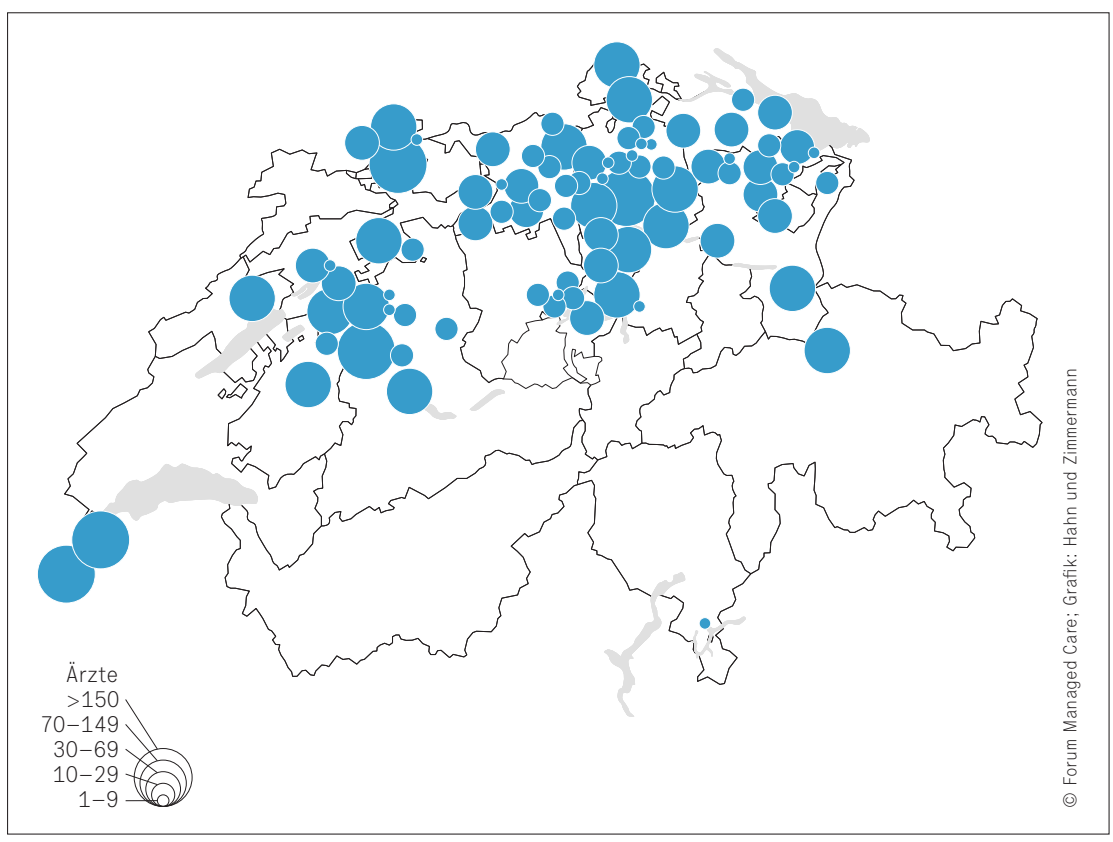

\section{Abbildung 4}

Ärztenetze pro Kanton mit Anzahl der im Netz betreuten Versicherten und Netzalter nach Betriebsjahren.

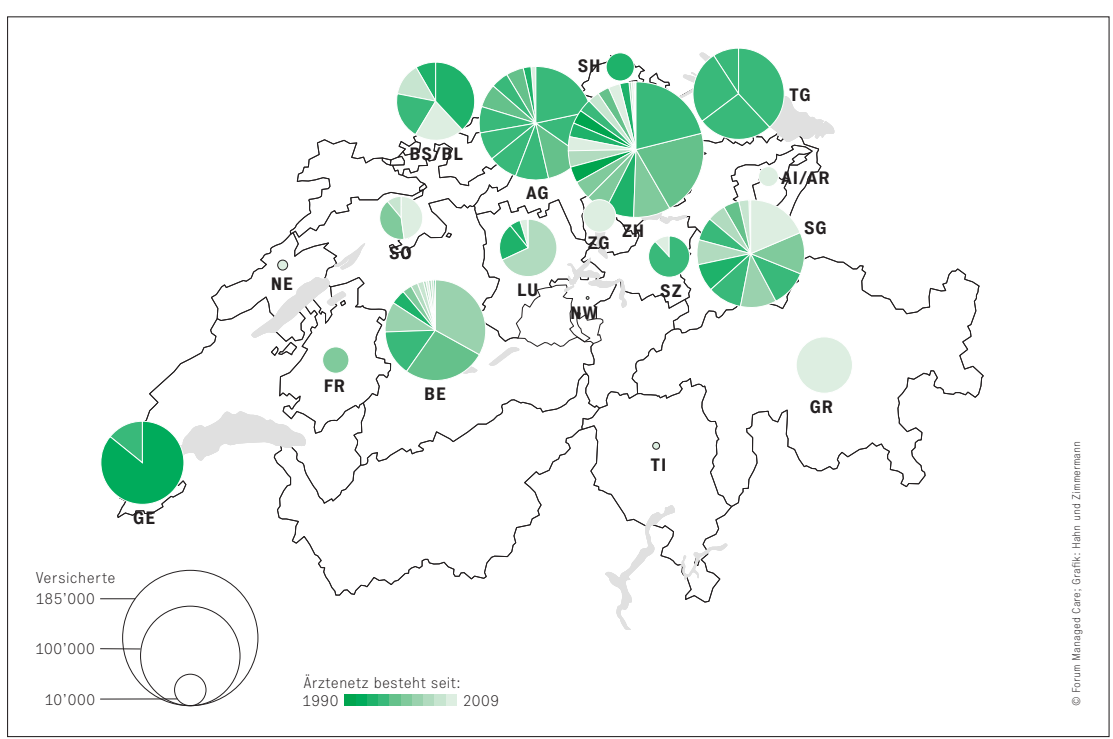

vereinbart, d.h. die Mitverantwortung für eine bestimmte Gruppe von Patienten, ein vereinbartes Kostenziel einzuhalten.

\section{Eindrückliche Qualitätsarbeit}

Beinahe alle Netze haben ein oder mehrere Elemente netzweiter Qualitätsarbeit implementiert: Schwerpunkt sind die Qualitätszirkel, die 96\% der Netze verbindlich durchführen (Abb. 5). Der Medianwert der obligatorischen Zirkel liegt bei 8 pro Jahr. Diese Ergebnisse liegen höher als jene aus der Pilotphase des FMHProjekts Q-Monitoring, wo drei Viertel der teilnehmenden Hausärzte eine Teilnahme an Qualitätszirkeln deklariert haben [3]. Weitere Elemente sind Critical Incident Reporting (CIRS, 53\%), Behandlungsleitlinien (41\%), vertraglich vereinbarte Offenlegung von Qualitäts- und/oder Kostendaten (55\%). Mehr als die Hälfte der Netze weist die Qualitätsarbeit durch einen regelmässigen Bericht zuhanden externer Stakeholder (43\%) und/oder durch ein Q-Zertifikat wie z.B. EQUAM, GMP (40\%) aus (Abb. 5, alle folgenden Abbildungen auf der nächsten Seite).

\section{Weitreichende und verbindliche Kooperationen}

Beinahe die Hälfte der Netze (43\%) verfügen über vertraglich geregelte Kooperationen mit anderen (externen) Leistungserbringern, insbesondere mit Spitälern (36\%), Notfalldiensten (33\%) und Call Centern (19\%) (Abb. 6). Ebenfalls rund die Hälfte der Netze (51\%) arbeiten mit sogenannten «Preferred Providern», d.h. bevorzugten Leistungserbringenden, denen sie ihre Patienten primär zuweisen: Am häufigsten sind dies Spezialärzte (45\%), Spitäler (35\%), Physiotherapien und Radiologie-Institute (je $27 \%$ ) sowie Labor (23\%) (Abb. 7). Ausgewählt werden diese «Preferred Provider» aufgrund persönlicher Erfahrungen (45\%) und/ oder Ergebnissen aus den Qualitätszirkeln (44\%), aber auch aufgrund von Qualitätsdaten (31\%) und Patientenangaben (28\%) (Abb. 8).

Der grundsätzliche Unterschied zwischen reinen Gatekeeping-Systemen und Integrierter Versorgung ist eine verbindliche Kooperation über mehrere Versorgungsebenen, d. h. eine «vertikale Integration» [4]. In diesem Sinne sind unsere Ergebnisse bedeutsam und widersprechen der geläufigen Meinung, dass die Schweizer Ärztenetze ausschliesslich Grundversorgung anbieten. Bei rund der Hälfte der Ärztenetze ist somit die Grundvoraussetzung für Integrierte Versorgung und deren Weiterentwicklung gegeben.

\section{Auf dem Sprung zu Integrierter Versorgung}

Die vorliegenden Resultate zeigen die beeindruckende Entwicklung der Ärztenetze und der Integrierten Versorgung in der Schweiz: Bis zu ein Drittel der Versicherten und rund die Hälfte der Grundversorger sind in 86 Ärztenetzen, vier Fünftel davon mit Budgetmitverantwortung. Doch das ist nur eine Dimension der In- 


\section{Abbildung 5}

Einrichtungen zur Qualitätsoptimierung in Ärztenetzen (in Prozent der Netze).

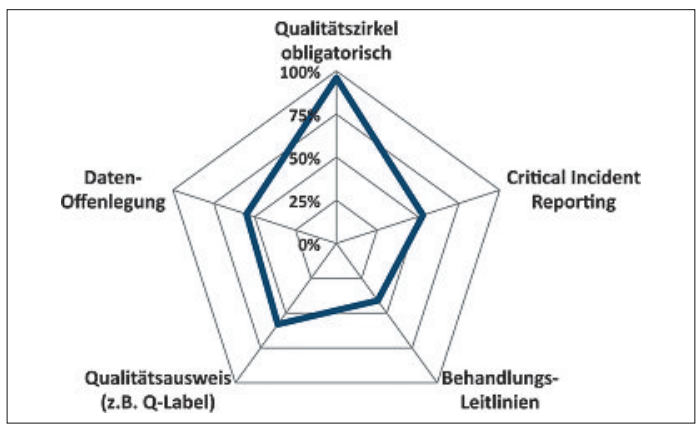

Abbildung 7

Preferred Provider, denen die Netze ihre Patienten primär zuweisen (in Prozent der Netze).

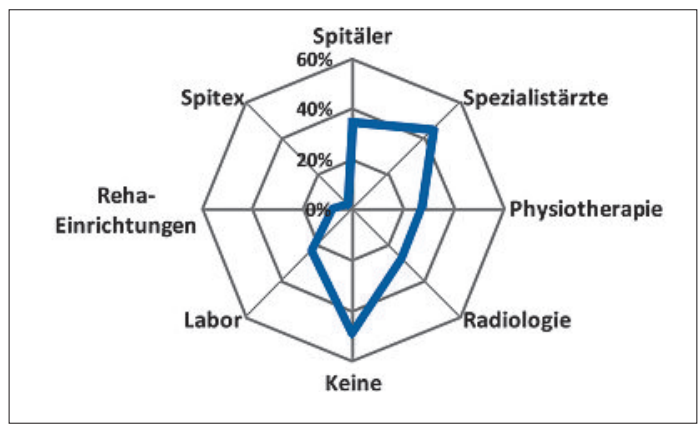

tegration. Für die Zukunft bedeutender ist die zweite, «vertikale» Dimension der Integration, nämlich die Kooperationen mit vor- und nachgelagerten Leistungsanbietenden sowie Preferred Providern, die heute schon bei vielen Netzen aufgebaut werden. Preferred Provider bzw. bevorzugte Kooperationen machen deutlich, um was es hier im Kern geht: Nicht die Einschränkung der freien Arztwahl ist das Wesentliche, sondern die Betreuung in einem Netz bevorzugter und deshalb aufeinander abgestimmter Leistungsanbieter.

\section{Abbildung 6}

Vertraglich geregelte Kooperationen mit externen Leistungserbringern (in Prozent der Netze).

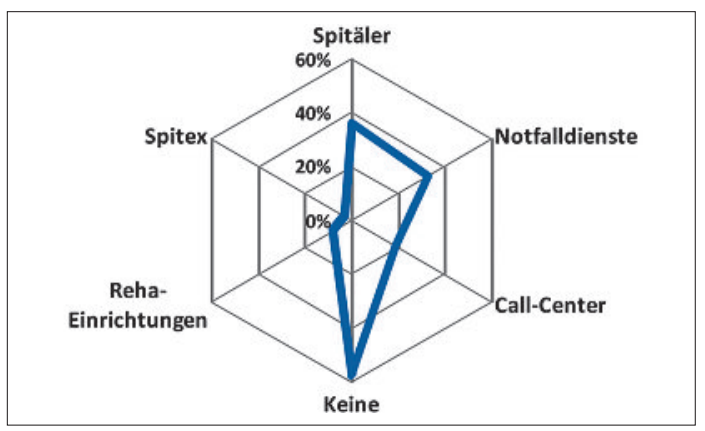

\section{Abbildung 8}

Kriterien, aufgrund derer die Preferred Provider ausgewählt wurden (in Prozent der Netze).

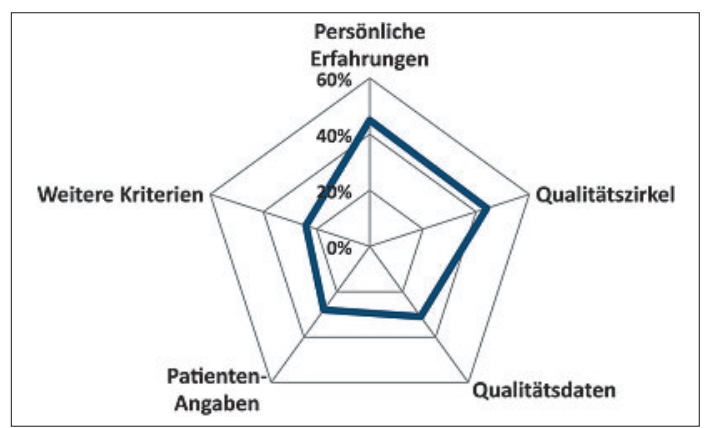

Literatur

1 Berchtold P, Peier K, Peier C. Erfolgreiche Entwicklung der Ärztenetze in der Schweiz. Schweiz Ärztezeitung. 2008;89(47):2038-41.

2 www.fmc.ch/infothek/aerztenetzeschweiz/

3 Nikolic V, Herren D. Die Ärzteschaft sichert Qualität und wie! Schweiz Ärztezeitung 2010;91(25): 973-6.

4 Strandberg-Larsen M, Krasnik A. Measurement of integrated healthcare delivery: a systematic review of methods and future research directions. Int J Integrated Care. 2009; Feb. 4:1. 